\title{
Effects of non-homogeneous flow on ADCP data processing in a hydroturbine forebay
}

\author{
S.F. Harding, M.C. Richmond*, P. Romero-Gomez, J.A. Serkowski \\ Hydrology Group, Pacific Northwest National Laboratory, Richland, WA, 99352
}

\begin{abstract}
Accurate modeling of the velocity field in the forebay of a hydroelectric power station is important for both power generation and fish passage, and is able to be increasingly well represented by computational fluid dynamics (CFD) simulations. Acoustic Doppler current profiler (ADCP) are investigated herein as a method of validating the numerical flow solutions, particularly in observed and calculated regions of nonhomogeneous flow velocity. By using a numerical model of an ADCP operating in a velocity field calculated using CFD, the errors due to the spatial variation of the flow velocity are quantified. The numerical model of the ADCP is referred to herein as a Virtual ADCP (VADCP).

Two applications of the VADCP are modeled in the numerical analyses presented. Firstly the virtual measurement error of the VADCP is calculated for a single instrument adjacent to the short converging intake of a powerhouse. Secondly, the flow discharge through the forebay is estimated from a transect of VADCP instruments at different distances from the powerhouse. The influence of instrument location and orientation are investigated for both cases.

A velocity error of up to $94 \%$ of the reference velocity is calculated for a VADCP modeled adjacent to an operating intake and is shown to decrease with distance from the powerhouse. Qualitative agreement is observed between the calculated VADCP velocities and reference velocities by a horizontal offset distance of $18 \mathrm{~m}$ upstream of the powerhouse.
\end{abstract}

Keywords: Acoustic Doppler Current Profiler (ADCP), Non-homogeneous velocity, hydropower, turbine, intake, virtual instrument

\section{1. Introduction}

Computational fluid dynamics (CFD) is a valuable approach to understand the flow characteristics in

${ }^{*}$ Corresponding author

Email address: marshall.richmond@pnnl.gov (M.C. Richmond)

Preprint submitted to Journal of Flow Measurement and Instrumentation

April 15, 2015 
and engineering design features can be readily evaluated [1. As computational resources are increased, the complexity of the numerical methods and detail of CFD solutions are also advancing.

Validation of the CFD results using field measurements is an important process to insure that the model represents the real flow conditions to an acceptable level. One field measurement technique available for this purpose is acoustic Doppler velocimetry, which provides velocity measurements throughout the water depth in an unobtrusive and relatively simple set-up through the use of an Acoustic Doppler Current Profiler $(\mathrm{ADCP})$.

ADCPs represent an increasingly popular flow measurement technique. One of the principle advantages for this technology is the ability to simultaneously measure three-dimensional flow velocities over the water depth in a number discretized sample bins. This is achieved by the transmission of acoustic signals in a number of diverging directions from the instrument, which are reflected back towards the instrument from suspended particles in the water. The velocity vector in each of these acoustic beam directions is calculated through the frequency shift of the reflected acoustic signal as it is received at the instrument in a mono-static configuration. The beam-wise velocity components from three or more beams are often then transformed in a Cartesian coordinate system for convenience.

Previous studies comparing CFD simulations and ADCP measurements of the Bonneville 2 forebay identified significant regions of non-homogeneous flows near the intakes and forebay shores [2]. These nonhomogeneous flow features are of length-scales on the order of the beam separation distances of diverging beam ADCP, which range from 1-22 $\mathrm{m}$ at this site. It is therefore hypothesized that the implicit assumption of homogeneity in the conventional ADCP post-processing algorithms may have a significant influence on the discrepancies between the theoretical and measured flow velocities. To investigate, the simulated flow fields computed by the unsteady Reynolds-averaged Navier-Stokes (URANS) CFD simulation are probed in such a way as to represent the sampling geometry of an ADCP. These sampled velocities are combined using the ADCP post-processing algorithm for a four beam Janus configuration to calculate the three-dimensional velocity vector of the virtual ADCP (VADCP).

Examples of this instrument analysis technique can be found in a number of recent studies. These include the use of virtual instruments to study the response of ADCPs to large eddy structures [3] and non-homogeneous riverine and tidal flows [4, as well as the sensitivity of discharge calculations to ADCP instrument performance [5]. In a broader range of flow instrumentation techniques, the ability to calculate CFD flow fields with increasing accuracy has facilitated the performance analyses of virtual instruments including S-type Pitot tubes, ultrasonic flowmeters and point measurements by an acoustic Doppler velocimeters 6, 7, 8,

Though the results presented herein are for a selected forebay with site-specific flow conditions, the generalized formulation of the numerical VADCP model allows this approach to be applied to a wide range of forebays and operating configurations. 


\section{Method}

\subsection{Computational Fluid Dynamics}

The hydroelectric power station forebay modeled is Powerhouse 2 of the Bonneville Lock and Dam on the Columbia River is shown in Figure 1. The width of the forebay is $320 \mathrm{~m}$ at its widest point, with a maximum depth of $D=30.7 \mathrm{~m}$. There are 24 intake bays supplying eight Kaplan turbine units (3 intake bays per unit), each with a rated power of 66.5 MW, as well as two smaller 13.1 MW units.

The depth contours of the forebay show a relatively localized area of excavation upstream of the turbine intakes, as shown in Figure 2a. The lateral recirculation in the $x-y$ plane is shown in Figure $2 \mathrm{~b}$

The Bonneville 2 forebay and upstream section of the Columbia River were modeled with the commercial computational fluid dynamics (CFD) code, STAR-CCM+ v9 [9]. The model was discretized into 45.6M polyhedral cells with variable density over the domain. A low cell density was set up in the upstream portion of the forebay, whereas a high resolution representation of the turbine intakes was used to allow accurate modelling of the forebay discharge flow behaviour. This refinement coincided with the sampling locations of the VADCPs in the analysis. The CFD flow field solution used in this analysis was produced by the URANS version of the realizable $\kappa-\epsilon$ turbulent model. In the simulated operating condition ('Full Load Condition', as described in [10]), all the turbine units in Powerhouse 2 and two spillways were in operation, and these discharges were known. Thus, those operating units were defined as outflows in the CFD model. The inflow was represented as a constant pressure boundary. The water surface was set up as a symmetry plane. The remaining boundaries (bathymetry, turbine intakes, spillway structures, etc.) were represented as no-slip walls. Wall functions were used on the latter boundaries, with the "two-layer" approach that allowed for the turbulent quantities $-\kappa$ and turbulent viscosity - to also be modeled at the walls, as opposed to the "single-layer" function, which does not include them.

The segregated solver was advanced at 2-second intervals. The convection term was $2^{\text {nd }}$-order upwind, and the temporal discretization was $2^{\text {nd }}$-order, allowing 20 inner iterations at each time step. The velocity field was averaged for $20 \mathrm{~min}$ after a "warm up" period of $40 \mathrm{~min}$. This mean field was used to conduct the analysis of the VADCP performance. During the transient simulation, strong flow recirculations were present at both sides of the powerhouse. Despite their highly dynamic behavior, these recirculations had little influence on the calculated VADCP velocities because they occurred in the shallow shoreline waters where the spread of the acoustic beams were considerably less than the scales of non-homogeneity.

ADCP measurements are available for that site at the simulated operating condition, and comparisons can be found in a publicly available report [2]. In the present study, we tested the sensitivity of the aforementioned flow solution ("base solution") to three settings: the averaging period, the time step, and the mesh size. We tested one parameter at a time. For the averaging period, we computed the field means over 60 min (20-min in base solution). For the time step, we set up $\Delta t=4 \mathrm{~s}(\Delta t=2 \mathrm{~s}$ in base solution), 


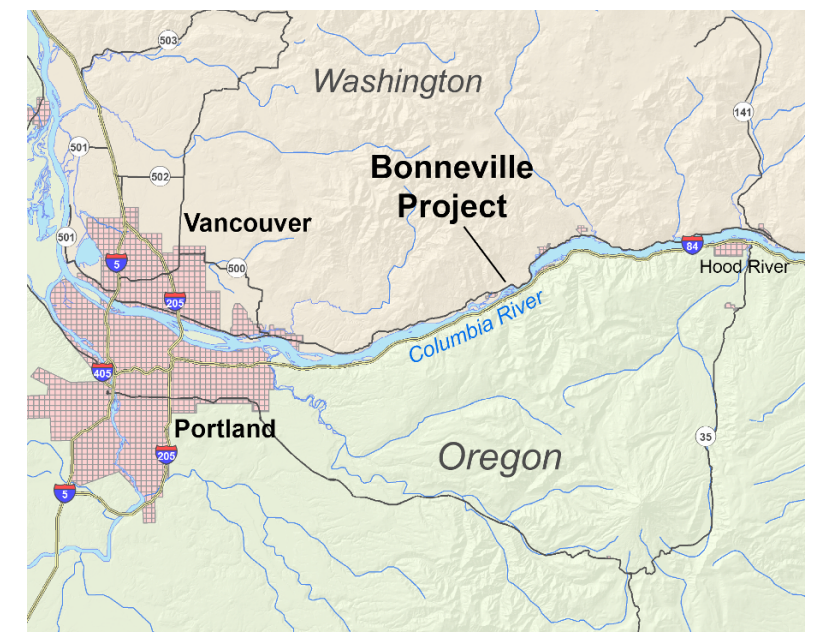

(a)

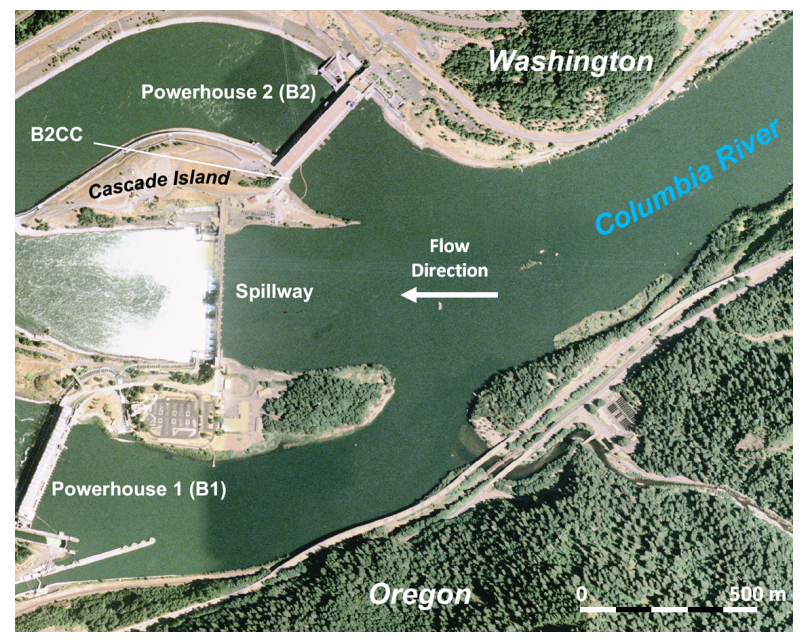

(b)

Figure 1: Location of Bonneville Lock and Dam on the Columbia River in the Pacific Northwest region of the USA (left) and detailed view of dam layout (right). Flow is from right to left in both images.

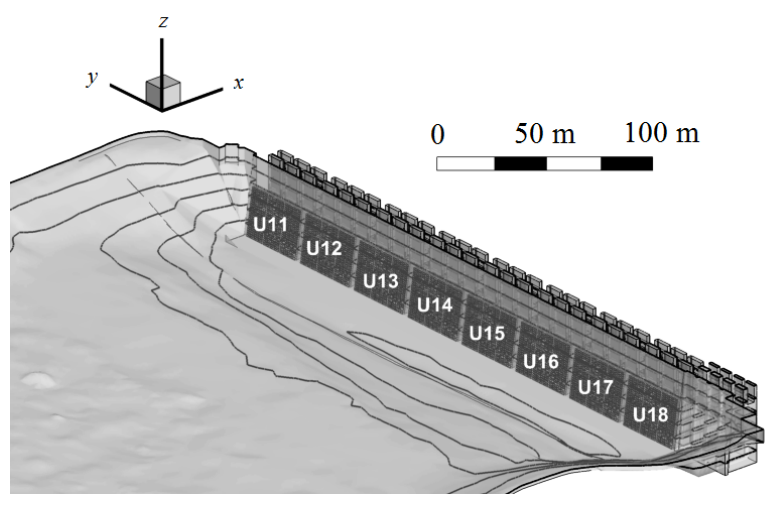

(a) Isometric view of Bonneville 2 powerhouse forebay and eight turbine intakes. Depth contour lines are shown in $5 \mathrm{~m}$ depth increments from the water surface. The local coordinate system definition and origin are also included in the top left corner.

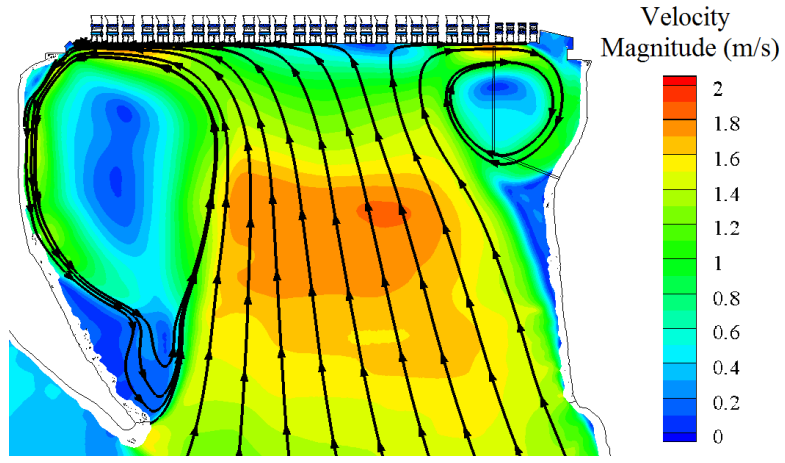

(b) Contour plot of velocity magnitude at $z=-2.0 \mathrm{~m}$ showing regions of recirculation with 10 stream-lines spaced equally at the entrance to the forebay.

Figure 2: Bathymetry and velocity vectors of the Bonneville 2 forebay. 
ran the simulation for $40 \mathrm{~min}$, and then collected the mean field for $20 \mathrm{~min}$, as in the base solution. For the mesh size, we coarsened the mesh to $15.3 \mathrm{M}$ cells $(45.6 \mathrm{M}$ in base solution), ran the coarse model for one hour, and collected the field mean for $20 \mathrm{~min}$. For the region of the forebay that was sampled by the VADCP, this coarsening represents an approximately uniform increase in mesh size by a factor of $\sim 1.4$. In comparison to the base solution, the test solutions showed limited effect of the selected parameters in terms of the mean absolute errors (MAE) of the velocity magnitude at the ADCP locations indicated in the report [10. MAE values were $0.01 \mathrm{~m} / \mathrm{s}, 0.03 \mathrm{~m} / \mathrm{s}$ and $0.07 \mathrm{~m} / \mathrm{s}$ for the averaging period, the time step, and the mesh size test solutions, respectively. These values were considered small in comparison to the mean velocity magnitude of $0.90 \mathrm{~m} / \mathrm{s}$ calculated from all the ADCP locations in the base solution. Therefore, we considered the base solution to suffice for describing the flow field in support of the present application of virtual ADCP performance assessment.

The coordinate system used in the following analyses is presented in Figure 2a, where $x=0$ at the powerhouse intake, the $y$-direction is aligned with the axis of the dam and the $z$-direction is positive vertically upwards from the mean water level.

\subsection{Virtual Acoustic Doppler Current Profiler Model}

\subsubsection{VADCP Methodology}

The virtual ADCP instrument is modeled by extracting velocity values from the CFD models at locations which correspond to the center of each bin of each acoustic beam. The velocities at equivalent bin heights along the central axis of the instrument are also recorded as this is the notional position of the virtual velocity measurement. These velocities represent the reference velocities of the VADCP, which are used to assess the accuracy of the velocities calculated by the VADCP model.

The velocity processing of the VADCP is calculated in the following steps:

1. The location, blanking distance, bin length and orientation of the virtual instruments are specified. In the analysis presented herein, the VADCP geometry represents a 4-beam ADCP configuration. The blanking distance to the bottom of the first bin is $0.875 \mathrm{~m}$ and the bin length is $0.25 \mathrm{~m}$, such that the depth profile is sampled in $0.25 \mathrm{~m}$ increments from a distance of $1.0 \mathrm{~m}$ from the instrument.

2. The velocity vectors are extracted from the time-averaged CFD solution at the locations of each bin center of the four diverging acoustic beams. The velocity vectors are also extracted from the CFD velocity field at locations along the axis of the instrument at each bin depth, to be used as a reference velocity.

3. The velocity vectors are converted from Cartesian coordinates into beam-wise velocity vectors. Letting the mean velocity vector of beam $i$ at bin $j$ be defined as $a_{i, j}=\left[U_{i, j}, V_{i, j}, W_{i, j}\right]$ and the unit beam 
direction vector be denoted as $\hat{d}_{i}=\left[\Delta x_{i}, \Delta y_{i}, \Delta z_{i}\right]$, then the component of the velocity vector in the direction of the acoustic beam is calculated by Equation 1 .

$$
b_{i, j}=a_{i, j} \cdot \hat{d}_{i}
$$

4. The beam-wise velocity vectors are combined to calculate a three-dimensional velocity vector in the instrument coordinate system. Defining the flow speed in the direction of the beam as positive velocity being towards the instrument, the coordinate transformation from beam coordinates to instrument coordinates is given by Equations $2 \mathrm{a}$-c. This coordinate transform has been modified from literature for the coordinate system described in Figure 3 11, 12. The angle of divergence of each acoustic beam from vertical is taken as $\theta=20^{\circ}$ in this analysis, and $c$ is used as a subscript to denote that the value is a calculated result of the coordinate transform.

$$
\begin{aligned}
u_{c}^{\prime} & =\frac{b_{1}-b_{2}}{2 \sin \theta} \\
v_{c}^{\prime} & =\frac{b_{3}-b_{4}}{2 \sin \theta} \\
w_{c}^{\prime} & =-\frac{b_{1}+b_{2}+b_{3}+b_{4}}{4 \cos \theta}
\end{aligned}
$$

5. The velocity vector is transformed from the instrument coordinate system to the global coordinate system using the roll $(\psi)$, pitch $(\vartheta)$ and yaw $(\phi)$ angles specified in the ADCP orientation input matrix. The Euler angle matrix transformation is given in Equation 3 .

$$
R=\left[\begin{array}{ccc}
\cos \psi & -\sin \psi & 0 \\
\sin \psi & \cos \psi & 0 \\
0 & 0 & 1
\end{array}\right]\left[\begin{array}{ccc}
\cos \vartheta & 0 & \sin \vartheta \\
0 & 1 & 0 \\
-\sin \vartheta & 0 & \cos \vartheta
\end{array}\right]\left[\begin{array}{ccc}
1 & 0 & 0 \\
0 & \cos \phi & -\sin \phi \\
0 & -\sin \phi & \cos \phi
\end{array}\right]
$$

The relationship between the velocity components in the instrument coordinate system and those in the global coordinate system is then given in Equation 4.

$$
\left[\begin{array}{l}
u_{c} \\
v_{c} \\
w_{c}
\end{array}\right]=R\left[\begin{array}{c}
u_{c}^{\prime} \\
v_{c}^{\prime} \\
w_{c}^{\prime}
\end{array}\right]
$$

6. The calculated velocity vectors are compared with the co-located CFD velocity vectors to perform an error analysis. The virtual measurement error of the VADCP is calculated by Equation 5 by comparing the calculated velocities against the reference velocities at the equivalent axial distance 


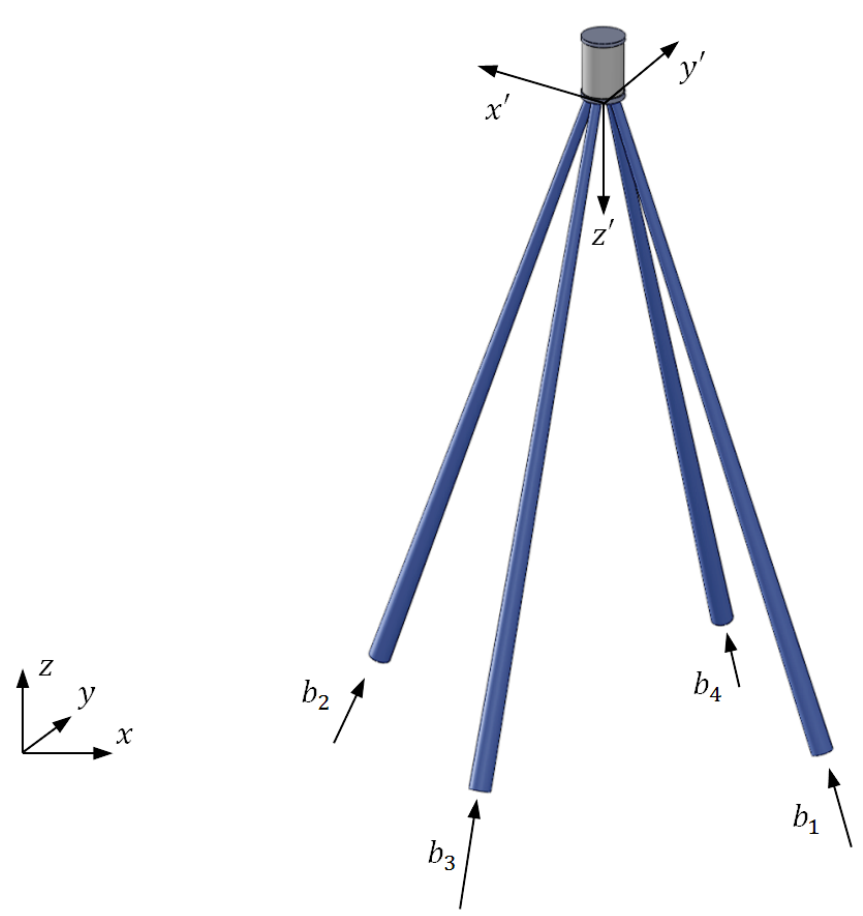

Figure 3: Beam numbering scheme for VADCP in default orientation

$$
\left[\begin{array}{l}
u_{e} \\
v_{e} \\
w_{e}
\end{array}\right]=\left[\begin{array}{l}
u_{c} \\
v_{c} \\
w_{c}
\end{array}\right]-\left[\begin{array}{l}
u_{r} \\
v_{r} \\
w_{r}
\end{array}\right]
$$

As discussed in Section 2.1, the velocities used in the following analyses are time averaged over a period of 20 minutes. The mean velocities are denoted by the upper-case form of the nomenclature described in this section. Because the velocity transform from beam coordinates to global coordinates is linear, the velocity error calculated using the 20 minute time averaged velocity field is equivalent to the mean error of a time dependent analysis.

Note that this process models the effect of using four separate beam location to infer a single velocity measurement. The significant challenge of holding station for the duration required to calculate a stable mean velocity in a vessel-mounted field measurement is not accounted for. Also, the effect of Doppler noise, seeding material concentrations, instrument fouling with debris, signal interference of boundaries, and other issues associated with field deployments of acoustic Doppler instruments have been neglected. In this way the implications of the post-processing algorithm assumptions can be studied in isolation. 


\subsubsection{Generalized Application of VADCP Model}

The method described in Section 2.2.1 can be implemented in a range of flow conditions generated using a number of CFD modeling techniques [3, 4, 5. The following key assertions should be applied when implementing the VADCP methods described in the present analysis:

Beam naming convention: The assignment of beam velocities is not unique and alternative velocity transformations to Equation 2 are possible. As such, the beam allocation defined in Figure 3 must be followed.

Coordinate system orientation: The orientation of the Cartesian coordinate system of the flow field must be defined relative to the instrument coordinate system defined in Figure 3 Right-hand coordinate system conventions have been used throughout, in both the coordinate system axis definitions and Euler angle matrix notation.

Bathymetric interference: Simultaneous beam-wise velocities are required in the coordinate transformation of Equation 2. Due to the diverging nature of the beam, one or more of the same location may no longer exist within the fluid volume of a CFD solution with irregular bathymetry. Such locations require alternative coordinate transforms which are not included in this analysis.

\subsection{Analysis Approach}

The analysis has been divided into two parts. Firstly, the performance of an individual VADCP is modeled in a range of positions adjacent to an operating intake.

Secondly, a number of VADCP instruments were modeled in transects of the forebay at a range of distances from the powerhouse to characterize the power station discharge. The discharge calculated using the derived VADCP velocities is compared against the actual discharge calculated using the reference velocities at the same nominal measurement points. Further details of these two analyses are presented below.

The VADCP is modeled in both upward-facing and downward-facing directions to represent bottommounted and vessel-mounted deployments, respectively. An ADCP deployed in a bottom mounted configuration typically requires the use of divers or a remotely operated vehicle (ROV) to control the yaw orientation. For vessel-mounted surface deployments, the instrument is aligned relative to the vessel. For ease of station keeping the vessel is typically aligned into the flow direction. In this way, the yaw orientation of the downward-facing instrument is able to be controlled much less expensively than the upward-facing configuration.

Again, the results are site specific as a result of the bathymetry, configuration and operating conditions of the forebay. However, the VADCP analysis presented herein demonstrates how the results of this generalized method can be interpreted as a useful and informative tool in the planning of a range of real ADCP deployments. 


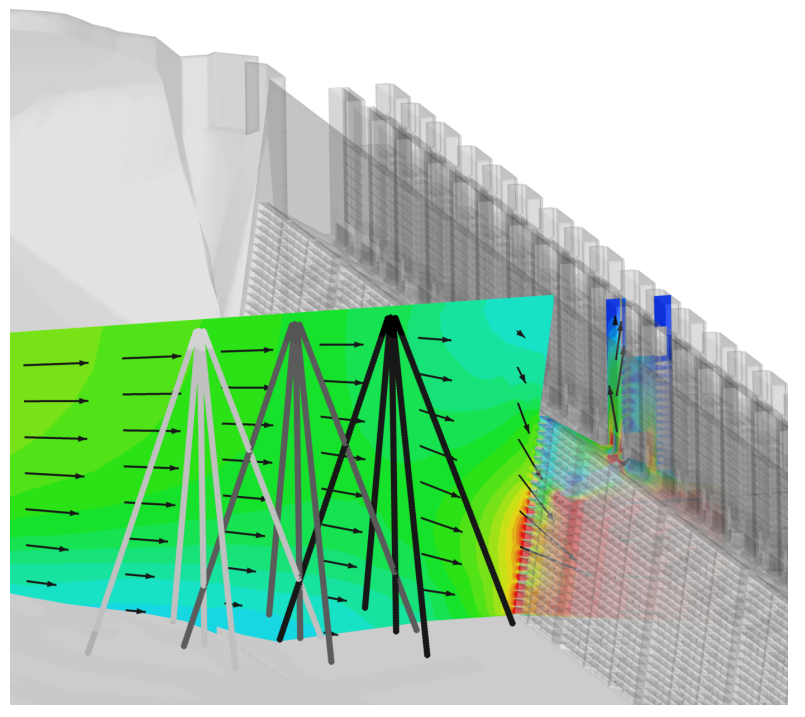

(a) Downward-facing VADCPs

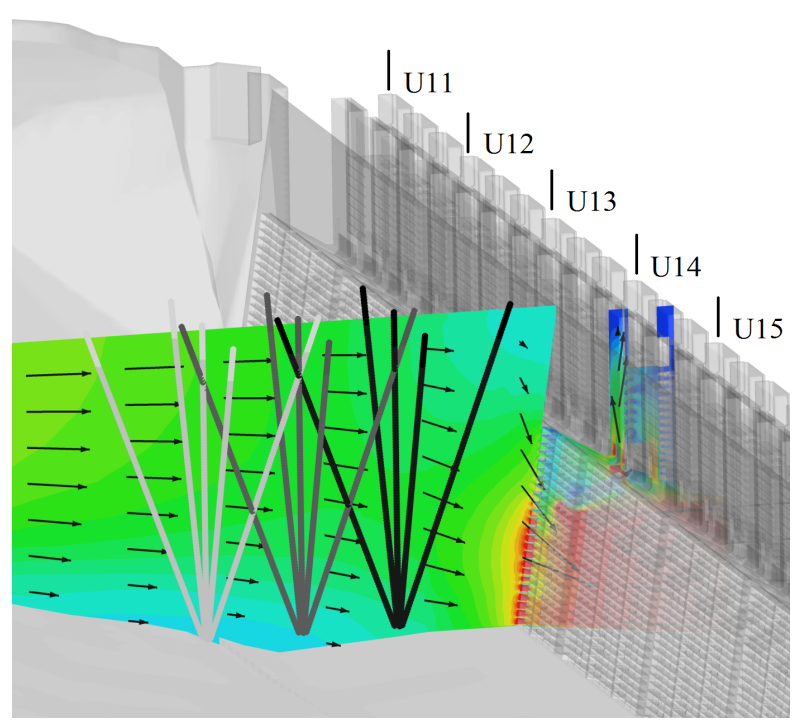

(b) Upward-facing VADCPs

Figure 4: Contour slice of instantaneous velocity magnitude in the vertical plane, showing the beams of a VADCP adjacent to powerhouse intake. The black lines indicate the beams of the VADCP in the nominal location directly adjacent to the intake. The dark grey and light grey beams show the instrument position with the offset of $\Delta x / H=-0.5$ and $\Delta x / H=-1$, respectively

\subsubsection{Individual VADCP Analyses}

The accuracy of a virtual point velocity measurement by a VADCP in the vicinity of an operational turbine intake is explored in this section. This study is designed to asses the appropriateness of the use of an ADCP to validate the numerical solution of the CFD in the powerstation forebay.

A VADCP was modeled at the central intake bay of Unit 14 of the Bonneville 2 powerhouse (see Figure 2a). The default instrument configuration is in the downward-facing orientation, with the downstreammost beam reaching the forebay floor at the base of the intake. This location is denoted $\Delta x / H=0$. Defining the height of the intake as $H=18 \mathrm{~m}$, the effect of moving the instrument away from the intake with an offset of $\Delta x / H=-0.5$ and $\Delta x / H=-1$ were investigated as shown in Figure 4 a

Because of the diverging beam configuration, the spatial resolution of the measurement decreases with distance from the instrument. As such, an upward-facing, bottom-mounted VADCP was modeled at the same locations to observe the impact of the beam spread. The analysis was performed at the same three offset distances in the $x$-direction, as shown in Figure $4 \mathrm{~b}$, The deployment depth of the upward-facing VADCP was kept constant to provide consistency between the beam spread of the three instruments at a specific water depth, while also following the convention of the constant deployment depth of the downward-facing instruments.

In the default instrument orientation $\left(\phi=0^{\circ}\right)$, the horizontal component of Beam 1 of the VADCP is 

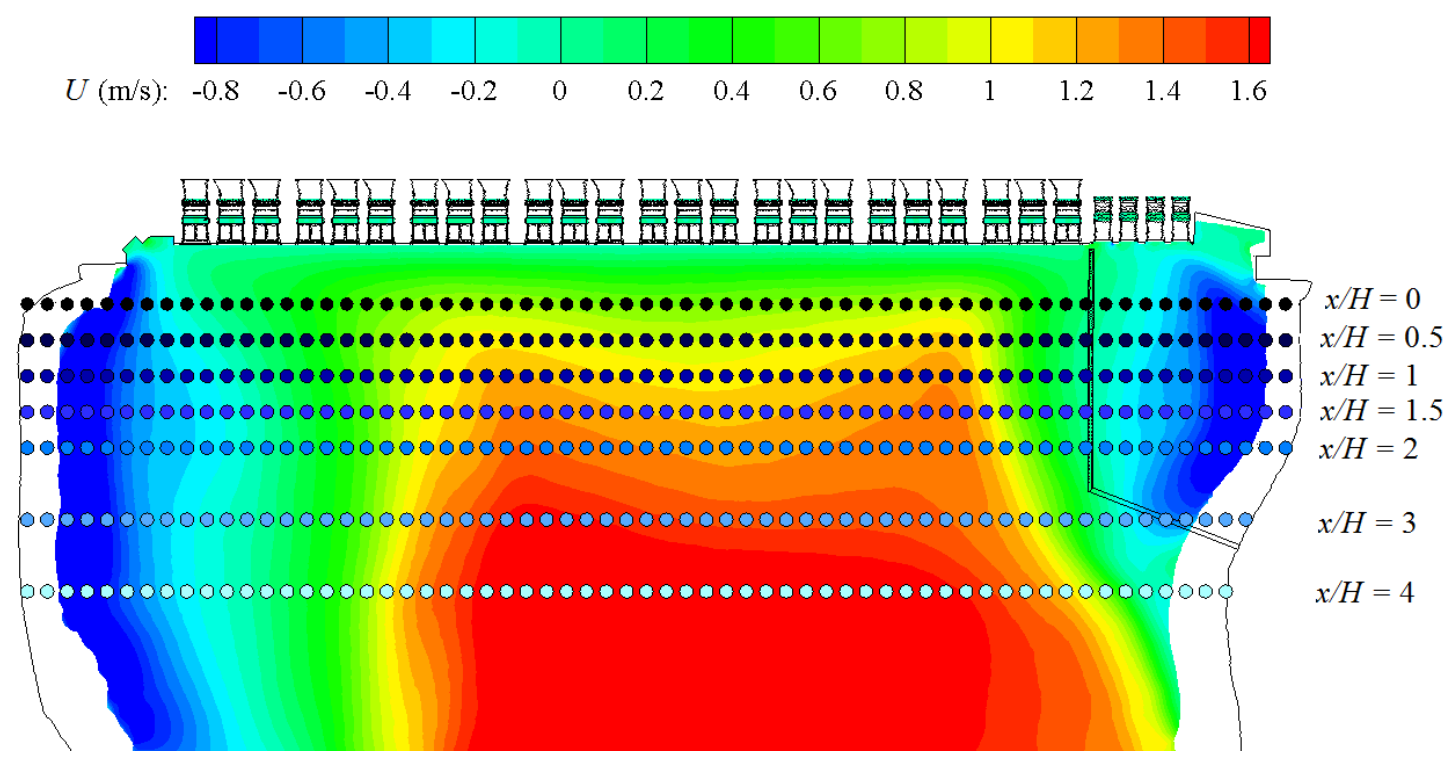

Figure 5: Location of VADCP transects in the forebay with contours of mean stream-wise velocity at $z=-5 \mathrm{~m}$. The black outline shows the perimeter of the forebay at $z=0 \mathrm{~m}$.

aligned with the $-x$ direction for all offset distances. In this orientation, $U_{c}$ is purely a function of $b_{1}$ and $b_{2}$ as the instrument and global coordinate systems are aligned.

The influence of yawing the instrument with an angle of $\phi=45^{\circ}$ is also presented. In this case, the calculated stream-wise velocity from the VADCP is a function of $b_{1}, b_{2}, b_{3}$ and $b_{4}$ through the Euler angle matrix transformation of Equation 3

\subsection{2. $V A D C P$ Transect Analyses}

A series of VADCP transects were modeled at a range of distances from the powerhouse. Only downwardsfacing instruments were considered for virtual discharge measurements of this nature are typically performed from boat-mounted units. As with the individual instrument analyses described in Section 2.3.1, the default $x$-location of the VADCP transect is the distance from the powerhouse where the downstream-most beam reaches the ground at the base of the intake. Transects at offsets of $x / H=[0,-0.5,-1,-1.5,-2,-3,-4]$ were simulated with an instrument spacing of $\Delta y=5 \mathrm{~m}$. A total of $61-65$ VADCPs were simulated for each transect, depending on the width of the forebay at the transect location, as shown in Figure 5 .

In the default orientation, the horizontal component of Beam 1 of the VADCP is aligned with the $-x$ direction for all offset distances. The effect of yawing the instrument by $\phi=45^{\circ}$ is also explored for each transect.

The discharge, $Q$, through the forebay is calculated by Equation 6 , when $U$ is perpendicular to the 
transect cross-section plane.

$$
Q=\int U \mathrm{dA}
$$

The cross-section of the forebay was discretized into rectangular and triangular areas of dA, with the virtual velocity measurements defining the node locations. The integral of Equation 6 was then solved numerically to calculate the discharge.

Regions of the flow around the periphery of the cross-section are unable to be measured by the diverging beams of ADCP instruments [13. These zones include:

- Top Zone: Unmeasured area of the instrument blanking distance

- Side Zone: Solid boundary contaminates acoustic signal in shallow water

- Bottom Zone: Contamination of the acoustic signal of one or more acoustic beams through side lobe reflections.

Therefore, when a total discharge measurement is required, the flow behaviour in these areas must be estimated. This is typically achieved by extrapolating the recorded velocities in the measurable area to the boundaries of the channel [14, 15, 16,

However accurate the method of approximating these unmeasurable region is, some level of error is introduced between the measured and actual velocity. The benefit of numerical modelling of a VADCP is that errors that are encountered in real deployments can be isolated and removed. For this reason, the discharge calculation herein only accounts for the flow in the measurable region and no extrapolation towards the boundary are considered. As such, the cross-sectional area used in the discharge calculation is limited by the location of the deepest available velocity measurement of each VADCP in the transect.

\section{Results}

\subsection{Individual VADCP Analyses}

At an offset of $\Delta x / H=-1$, both the downwards-facing and upwards-facing instruments calculate a stream-wise velocity component which agrees closely with the reference velocity. However as the instrument is moved towards the region of accelerated flow at the intake, the agreement between the calculated and reference velocity reduces, as shown in Figure 6. A maximum error of $94 \%$ of the depth-averaged reference flow velocity is calculated for the downward-facing instrument at $\Delta x / H=0$.

The error between the calculated and reference velocity is at its minimum closest to the device. At these locations, the divergent acoustic beam separation is relatively low such that the beam pairs sample similar flow conditions and the assumption of homogeneity can be applied. 

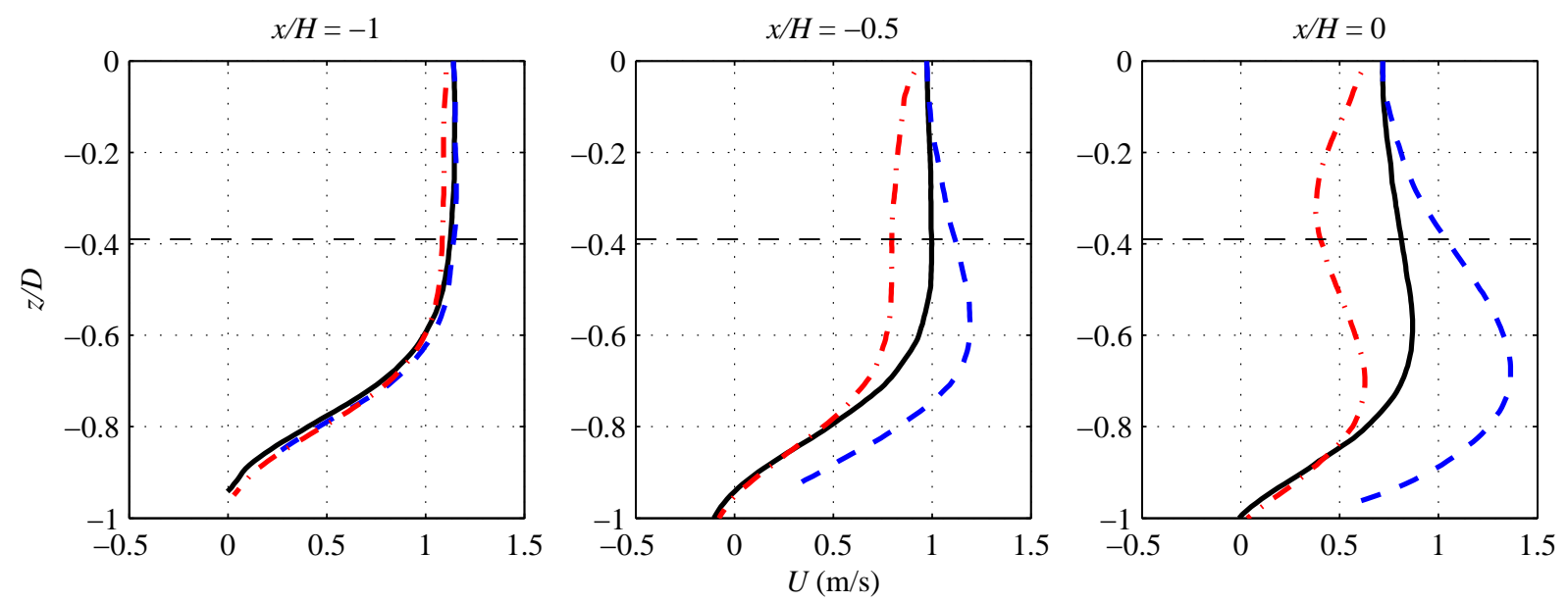

$U_{r}---U_{c}$ (Downward-facing) $:-\cdot \cdot U_{c}$ (Upward-facing)

Figure 6: Comparison of calculated VADCP velocities, $U_{c}$, with the reference velocity $U_{r}$ (black) as function of depth. The calculated velocities of the downwards-facing and upward-facing VADCP are shown in blue and red, respectively. The upper edge of the turbine intake is indicated with a dashed black line.

The beam-wise velocities of Beam 1 and Beam 2 are a function of both the stream-wise and vertical velocity components of the flow. Both of these velocity components are non-homogeneous, as shown by the vectors of Figure 4. The analysis defined in Section 2.2 was recalculated to determine the relative contributions of the $U$ and $W$ components to the error for the case of zero $x$-offset. In order to calculate the contribution of the non-homogeneous $U$-velocity component, the $W$-velocity component of each beam is set to equal the reference value of $W_{r}$ in the analysis. Similarly, the $U$-velocity component of each beam is set to equal the reference value of $U_{r}$ to determine the contribution of the non-homogeneous $W$-velocity component. The total error is the sum of these contributions, and is equal to the error calculated in the original analysis using the unmodified beam velocities, as defined in Equation 5. The velocity error is normalised by the depth-averaged reference velocity, $\overline{U_{r}}$, in the results presented in Figure 7

From Figure 7 it can be seen that the non-homogeneous vertical velocity is the primary source of the VADCP error over the majority of the water column for both instrument orientations.

A comparison of the VADCP velocity measurements with the yaw angles of $\phi=0^{\circ}$ and $\phi=45^{\circ}$ is presented in Figure 8. The black and blue lines of this figure are identical to that of Figure 6, showing the reference and calculated stream-wise velocity components for a downwards-facing VADCP. The red line shows the effect of yawing the instrument by $\phi=45^{\circ}$, to make use of all four beams in the calculation as discussed in Section 2.3.1. The rotation of the device also increases the distance of the sample volumes of the downstream-most beam from the accelerating flow at the intake. A significant difference between the two calculated velocities is only observed for the case of $\Delta x / H=0$, with a maximum error of $70 \%$ of the 

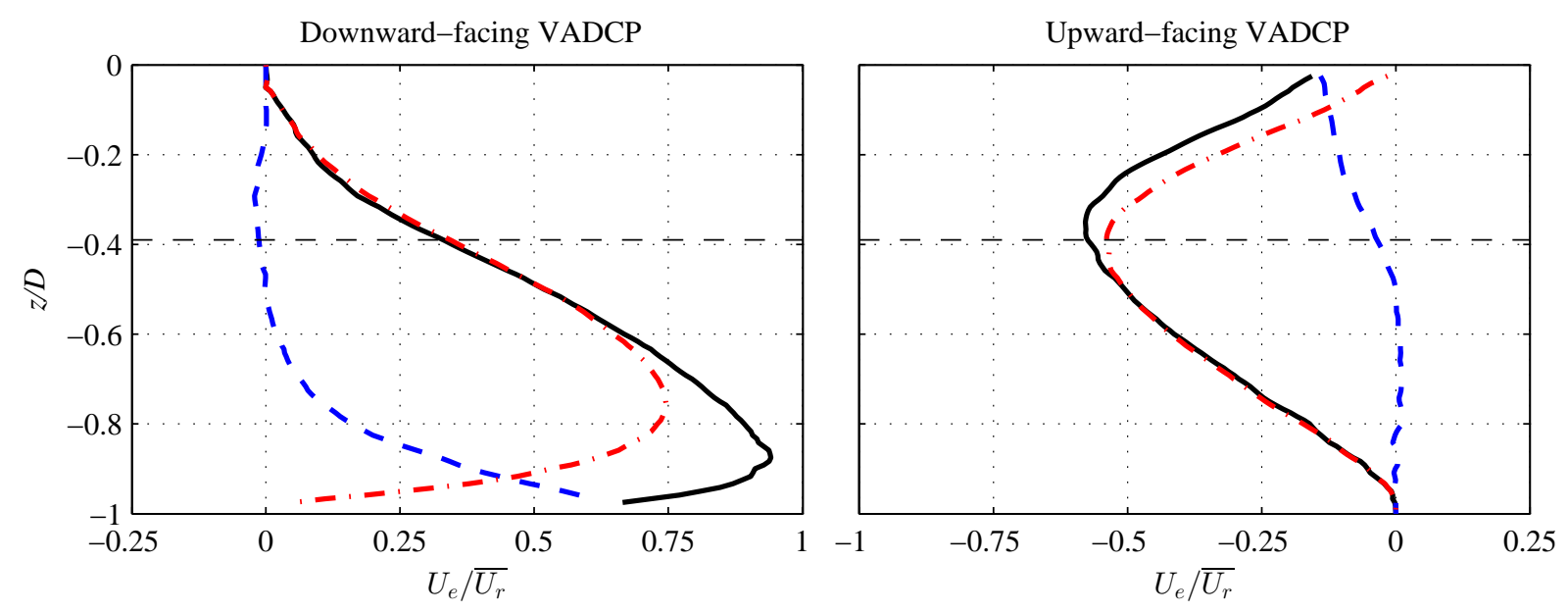

Total $---U$ contribution $\cdot-\cdot-W$ contribution

Figure 7: Relative contribution of the non-homogeneous $U$ and $W$ velocity components to the error of the calculated VADCP velocity. The left plot presents the results of the downward-facing orientation and the results of the upwards-facing device are shown on the right. The upper edge of the turbine intake is indicated with a dashed black line.

reference velocity. This is $24 \%$ less than the maximum relative error in the case where $\phi=0^{\circ}$.

\subsection{Discharge estimates through VADCP transects}

The discharge calculation is performed by numerical integration of Equation 6 for a number of transect distance from the powerhouse. Again, zero offset is defined as the $x$-location where the downstream beam intersects the base of the powerhouse and the forebay floor. The discharge is calculated using the reference stream-wise velocity and the calculated stream-wise velocity. The discharge error is calculated by Equation 7 and the results for each transect investigated are presented in Figure 9

$$
e_{Q}(\%)=100 \times\left(Q_{c}-Q_{r}\right) / Q_{r}
$$

The largest error in measurable discharge was calculated for the transect closest to the intake. The stream-wise velocity in the $y-z$ plane of this transect is shown in Figure 10. This figure shows that the primary source of the error is located at the intakes to the operating turbine units. This is the location of greatest localized flow acceleration as the water exits the large cross-sectional area of the forebay into the short converging intakes. This is therefore the location of the largest velocity difference between the acoustic beams. This discharge error is reduced to less than $10 \%$ by an offset of $|x / H|=0.5$.

The analysis presented herein is intended to be a general method that can be applied to any hydropower project, but the specific results from application of the method will depend on site specific conditions. 

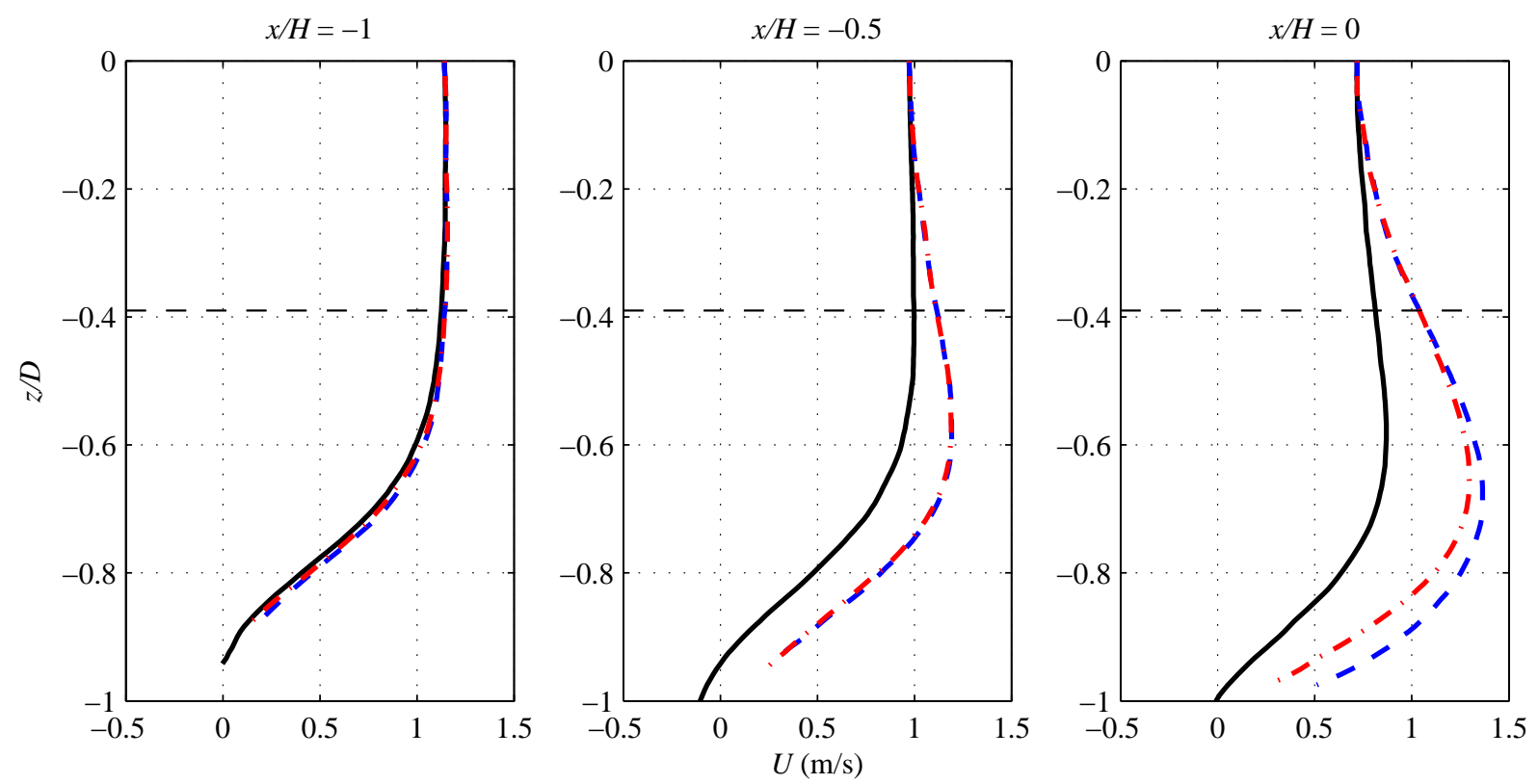

$$
U_{r}--U_{c}\left(\phi=0^{\circ}\right) \cdot-\cdot-\cdot U_{c}\left(\phi=45^{\circ}\right)
$$

Figure 8: Comparison of calculated VADCP velocities, $u_{c}$, with the reference velocity $u_{r}$ (black) as function of depth. The calculated velocities of the downwards-facing VADCP with yaw angle of $\phi=0^{\circ}$ and $\phi=45^{\circ}$ are shown in blue and red, respectively. The upper edge of the turbine intake is indicated with a dashed black line.

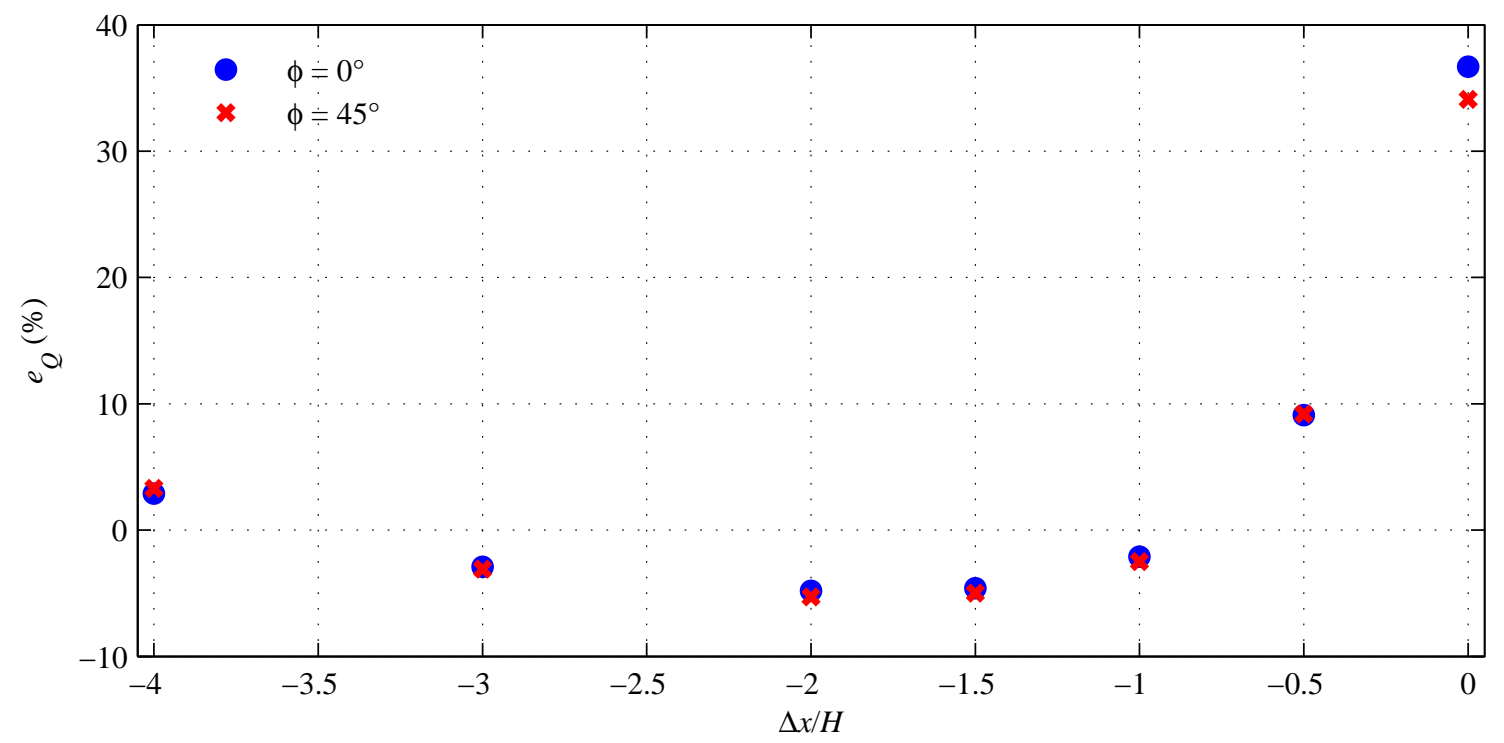

Figure 9: Comparison of discharge estimates of downward-facing VADCPs at different transect locations. 

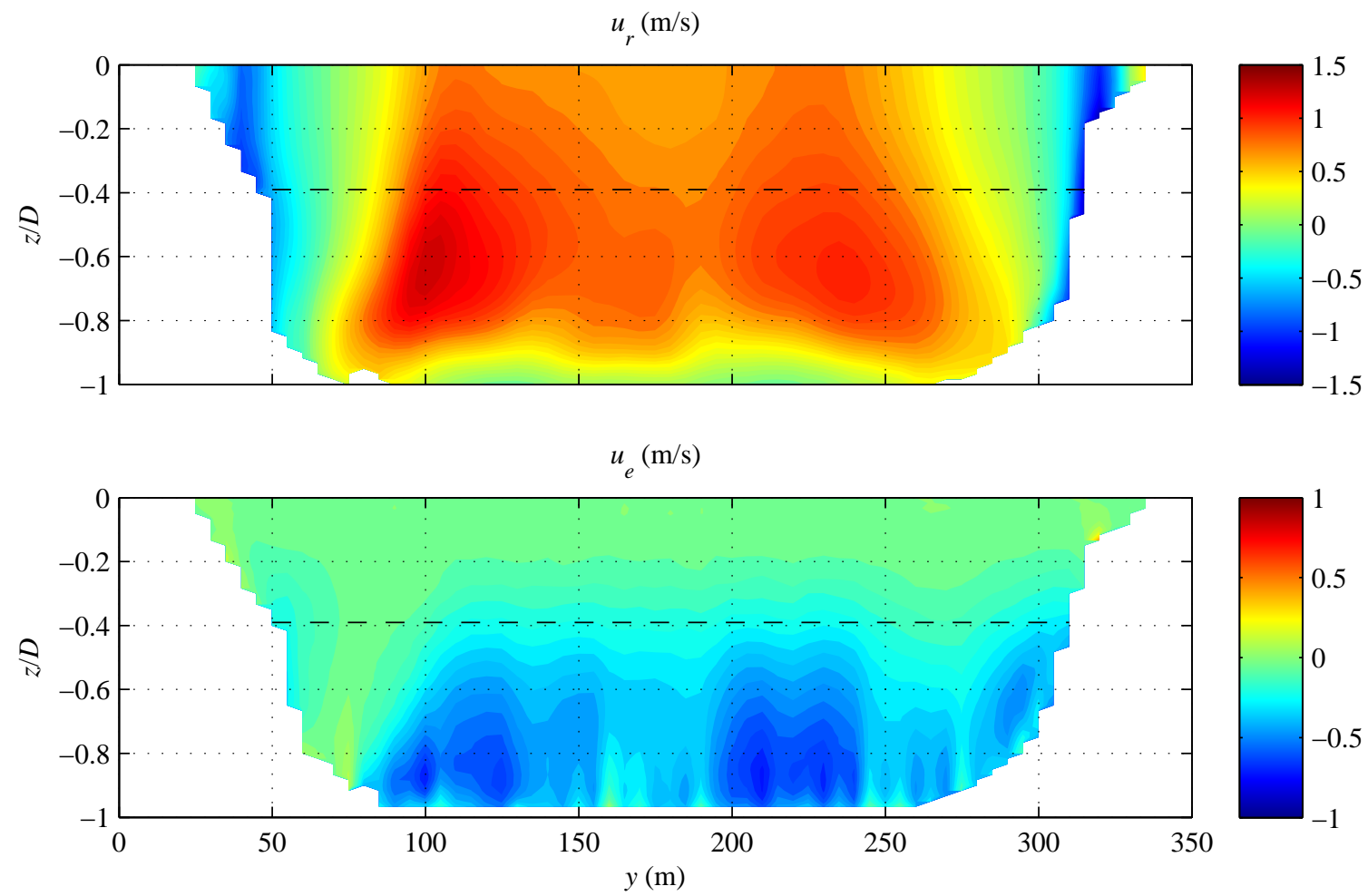

Figure 10: Spatial variation of the reference stream-wise velocity component (top) and measurement error (bottom) in the transect with offset $x / H=0$. The cross-sectional view is looking upstream from the powerhouse, and the upper edge of the turbine intakes are indicated with a dashed black line.

\section{Conclusions}

The flow velocities calculated using a virtual ADCP in the forebay of a short-converging intake hydropower station have been calculated for a range of instrument orientations and locations.

The acceleration of the flow into an operating turbine intake leads to non-homogeneous flows which invalidate the assumptions required to resolve three-dimensional velocities from the acoustic beams. Errors of up to $94 \%$ of the reference flow velocity were observed when the VADCP instrument was deployed directly in front of the intake. A reduction in the measured error was achieved by yawing the instrument by $\phi=45^{\circ}$ with the instrument in this position. These virtual measurement errors were significantly reduced by offsetting the instrument the distance of one intake height $(H=18 \mathrm{~m})$ upstream of the intake.

Similarly, errors in the virtual measurement of discharge were overestimated by up to $36.7 \%$ by a transect directly in front of the turbine intakes. This was reduced to less than $10 \%$ by offsetting the transect by one half of the intake height upstream of the powerhouse. The overestimation is further reduced to approximately $5 \%$ for transects at least one intake height upstream of the powerhouse.

While visible recirculation zones exist either side of the forebay, these are in relatively shallow water and 
therefore close to the water surface where the beams of the downward-facing VADCPs have not yet diverged to large separation distances. As such, these non-homogeneous flows do not contribute significantly to the error in calculated discharge.

Analyses of flow characteristics using CFD calculations are becoming increasingly useful tools in improving fish passage and turbine performance of hydropower projects. This virtual instrument analysis informs the efficient use of resources for a range of real field deployments to enable numerical flow solutions to be validated with ADCP instrumentation.

\section{Acknowledgements}

This research was funded by the U.S. Department of Energy, Energy Efficiency and Renewable Energy, Wind and Water Power Program.

Pacific Northwest National Laboratory (PNNL) is operated for the U.S. Department of Energy by Battelle under Contract No. DE-AC06-76RLO 1830.

Computations described here were performed using the facilities of the PNNL institutional computing center (PIC).

We also thank Laurie Ebner of the U.S. Army Corps of Engineers (USACE), Portland District for providing access to the Bonneville Dam forebay CFD model that was originally developed by PNNL under contract with USACE.

\section{References}

\section{References}

[1] C. L. Rakowski, L. L. Ebner, M. C. Richmond, Using CFD to Study Juvenile Salmon Bypass at Bonneville, Hydro Review 24 (1) (2005) 56-65.

[2] C. L. Rawkoski, M. C. Richmond, J. A. Serkowski, Bonneville Powerhouse 2 Fish Guidance Efficiency Studies: CFD Model of the Forebay, PNNL-21420.

[3] J. A. Boldt, Use of Numerical Simulations to Investigate the Performance of a Virtual Acoustic Doppler Current Profiler in Characterizing Flow, Masters, University of Illinois (2013).

[4] T. Tokyay, G. Constantinescu, J. A. Gonzalez-Castro, Investigation of Two Elemental Error Sources in Boat-Mounted Acoustic Doppler Current Profiler Measurements by Large Eddy Simulations, Journal of Hydraulic Engineering 135 (2009) $875-887$.

[5] G. Ramirez, A Virtual Flow Meter to Develop Velocity-index Ratings and Evaluate the Effects of Flow Disturbances on these Ratings, Masters, University of Illinois (2012).

[6] W. Kang, N. D. Trang, J. S. Shim, H. S. Jang, Y. M. Choi, Experimental and numerical investigations of the factors affecting the S-type Pitot tube coefficients, Flow Measurement and Instrumentation In Press.

[7] D. Zheng, P. Zhang, T. Xu, Study of acoustic transducer protrusion and recess effects on ultrasonic flowmeter measurement by numerical simulation, Flow Measurement and Instrumentation 22 (5) (2011) 488-493. 
[8] V. G. Gil Montero, M. Romagnoli, C. M. García, M. I. Cantero, G. Scacchi, Optimization of ADV sampling strategies using DNS of turbulent flow, Journal of Hydraulic Research (November) (2014) 1-8.

[9] CD-adapco, User Guide, STAR-CCM+ Version 9.04. CD-adapco, 2014.

URL http://www.cd-adapco.com

[10] ENSR, Bonneville Second Powerhouse Forebay ADCP Field Data Collection: 3697-003-106B, Tech. rep., ENSR Consulting \& Engineering (2000).

[11] A. Lohrmann, B. Hackett, L. P. Rø ed, High Resolution Measurements of Turbulence, Velocity and Stress Using a Pulseto-Pulse Coherent Sonar (1990).

[12] RD Instruments, ADCP Coordinate Transformation: Formulas and Calculations, Tech. Rep. July (1998).

[13] Teledyne RD Instruments, WinRiver Users Guide, Tech. Rep. February (2007).

[14] IEC, Field acceptance tests to determine the hydraulic performance of hydraulic turbines, storage pumps and pumpturbines, IEC-41.

[15] M. Simpson, Discharge Measurements using a Broad-Band Acoustic Doppler Current Profiler, Tech. rep., USGS, Sacramento, CA (2001).

[16] R. L. Gordon, Acoustic Measurement of River Discharge, Journal of Hydraulic Engineering 115 (7) (1989) 925-936. 